\title{
Cortical Biopsy Results in Alzheimer Disease: Correlation with Cognitive Deficits
}

\author{
E.M. Martin, R.S. Wilson, R.D. Penn, J.H. Fox, R.A. Clasen and S.M. Savoy
}

\begin{abstract}
This paper reports neuropsychologic and pathologic data for eleven patients with a clinical diagnosis of probable Alzheimer disease (AD) according to recently proposed NINCDS criteria. In all cases, the clinical diagnoses were verified by cortical biopsy using histopathologic criteria for definite AD. Similar tissue samples from nine non-demented autopsied patients were also evaluated and none received an histopathologic diagnosis of AD. Correlations between cortical plaque counts and neuropsychologic test scores are also presented. These data have important implications for 1) the accuracy of clinical diagnosis in AD; 2) the validity of NINCDS criteria for the clinical and pathologic diagnosis of $\mathrm{AD}$; and 3) the utility of cognitive test scores as indicators of disease severity.
\end{abstract}

RÉSUMÉ: Résultats de biopsies corticales dans la maladie d'Alzheimer: corrélation avec les déficits cognitifs. Cet article rapporte les données neuropsychologiques et pathologiques recueillies chez onze patients avec un diagnostic clinique de maladie d'Alzheimer (MA) probable, selon les critères NINCDS proposés récemment. Dans tous les cas, les diagnostics cliniques ont été vérifiés par biopsie corticale en se servant des critères histopathologiques caractéristiques de la MA. Des échantillons de tissus similaires provenant de l'autopsie de neuf patients non déments furent également évalués et aucun ne reçut un diagnostic histopathologique de MA. Les corrélations entre les décomptes des plaques corticales et les scores des examens neuropsychologiques sont aussi présentées. Ces données ont des implications importantes pour: 1) l'exactitude du diagnostic cliniques dans la MA; 2) la validité des critères NINCDS pour le diagnostic clinique et pathologique de la MA; 3) l'utilité des scores aux épreuves cognitives comme indicateurs de la sévéreité de la maladie.

Can. J. Neurol. Sci. 1986; 13:406 